\title{
The NP06/ENUBET experiment: a monitored neutrino beam
}

\author{
A. Branca ${ }^{1,2}$ for the ENUBET Collaboration* \\ ${ }^{1}$ Phys. Dep. Università di Milano-Bicocca, piazza della Scienza 3, Milano, Italy \\ ${ }^{2}$ INFN, Sezione di Milano-Bicocca, piazza della Scienza 3, Milano, Italy \\ E-mail: antonio.branca@mib.infn.it
}

The CERN NP06/ENUBET experiment is developing a new neutrino beam with unprecedented precision on the flux, flavor composition and energy of the produced electron and muon neutrinos. Such a goal will be accomplished by monitoring the charged lepton in kaon and pion decays. Therefore, the facility will be ideal for high precision neutrino cross-section measurement at the $\mathrm{GeV}$ Scale, a crucial aspect to boost the sensitivity of future next-generation long-baseline experiments and for the improvement of neutrino interaction models. In this paper, we present the current status of the project, focusing on the improved transfer line and proton target design, corresponding particle identification performance and first steps toward the assessment of systematics.

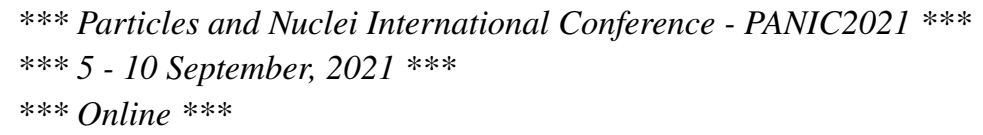

*F. Acerbi, I. Angelis, M. Bonesini, F. Bramati, A. Branca, C. Brizzolari, G. Brunetti, M. Calviani, S. Capelli, S. Carturan, M.G. Catanesi, S. Cecchini, N. Charitonidis, F. Cindolo, G. Cogo, G. Collazuol, F. Dal Corso, C. Delogu, G. De Rosa, A. Falcone, B.Goddard, A. Gola, F. Iacob, C. Jollet, V. Kain, B. Klicek, Y. Kudenko, Ch. Lampoudis, M. Laveder, A. Longhin, L. Ludovici, E. Lutsenko, L. Magaletti, G. Mandrioli, A. Margotti, V. Mascagna, N. Mauri, L. Meazza, A. Meregaglia, M. Mezzetto, M. Nessi, A. Paoloni, M. Pari, E.G. Parozzi, L. Pasqualini, G. Paternoster, L. Patrizii, M. Pozzato, M. Prest, F. Pupilli, E. Radicioni, C. Riccio, A.C. Ruggeri, D. Sampsonidis, C. Scian, G. Sirri, M. Stipcevic, M. Tenti, F. Terranova, M. Torti, S.E. Tzamarias, E. Vallazza, F. Velotti, L. Votano 


\section{NP06/ENUBET: a monitored neutrino beam}

The main source of systematic uncertainty on the neutrino cross-section comes from the knowledge of the initial flux in conventional neutrino beams. The idea behind ENUBET [1,2] is to realize the so called "monitored neutrino beams" [3], where a high controlled neutrino flux can be obtained by measuring the charged leptons from mesons along the decay tunnel. With this technique improvements of one order of magnitude in the neutrino cross-section precision are under reach, beneficial for the sensitivity of long-baseline experiments and neutrino interaction models.

ENUBET started as an ERC project in June 2016, with the purpose of developing a facility for the measurement of the large angle positrons from $K^{+} \rightarrow \pi^{0} e^{+} v_{e}$ decay, allowing to constrain the $v_{e}$ flux. Furthermore, ENUBET was approved as a Neutrino Platform experiment (NP06) at CERN in March 2019. In this context, ENUBET is exploring the possibility to monitor large angle muons from $K^{+} \rightarrow \mu^{+} v_{\mu}$ and $K^{+} \rightarrow \pi^{0} \mu^{+} v_{\mu}$, as well as muons from pion decays. Therefore, a constraint on the high and low energy $v_{\mu}$ component of the neutrino flux can be eventually set.

\section{Transfer line design and performance}

In ENUBET the pions and kaons produced by protons hitting a fixed target are focused and momentum selected, with a momentum bite of $O(5-10 \%)$, by a short transfer line (TL) of about $\sim 20 \mathrm{~m}$. The TL is designed for a $8.5 \mathrm{GeV} / \mathrm{c}$ particle beam, an optimal choice for the $e^{+} / \pi^{+}$ separation and to span the region of interest for the neutrino energy in DUNE and HyperK. The latest versions of the TL implement a double dipole configuration. The chosen configuration allows to reduce backgrounds and the $v_{e}$ component at the neutrino detector from early decays in the first part of the TL. A complete G4beamline [4] simulation is performed and allows to evaluate the beam composition. Results from the simulation show that an increase of the kaon flux at the tunnel entrance is obtained with respect to previous TL versions. The shielding of the TL components is included in the complete G4Beamline simulation, and has been optimized with FLUKA and GEANT4. A tungsten foil, with optimised thickness, is installed downstream the target to suppress the positron background.

Fig. 1-left shows the energy spectrum for $v_{e}^{C C}$ interactions in a 500 ton, $6 \times 6 \mathrm{~m}^{2}$ front face detector placed $50 \mathrm{~m}$ away from the tunnel end. A separation of the population of $v_{e}$ produced inside the tunnel from the ones from proton dump and first section of the TL is clearly visible. Above $1 \mathrm{GeV}$ more than $80 \%$ of the $v_{e}$ flux is produced inside the tunnel. The latest TL design allows to obtain an increase of $v_{e}^{C C}$ events of 2.5 with respect to previous versions, and a total of $10^{4} v_{e}^{C C}$ events in 2 years of operations are expected, assuming $4.5 \times 10^{19}$ POT/year at SPS.

A narrow band beam allows a precise estimate of the $v_{\mu}$ energy, exploiting its correlation with the distance $(\mathrm{R})$ from the beam axis of the neutrino interaction vertex ("narrow band off-axis" technique, see fig. 1-right). With the $8.5 \mathrm{GeV} / \mathrm{c}$ beam (DUNE optimized beamline) a 8-25\% energy resolution is achieved in the DUNE energy range and a $30 \%$ resolution in the HyperK one. An ongoing R\&D activity is developing a multi momentum beamline $(4.5,6$ and $8.5 \mathrm{GeV} / \mathrm{c})$ to populate and enhance resolution in the low energy region. Such beamline is then optimised for both, DUNE and HyperK region of interests. 

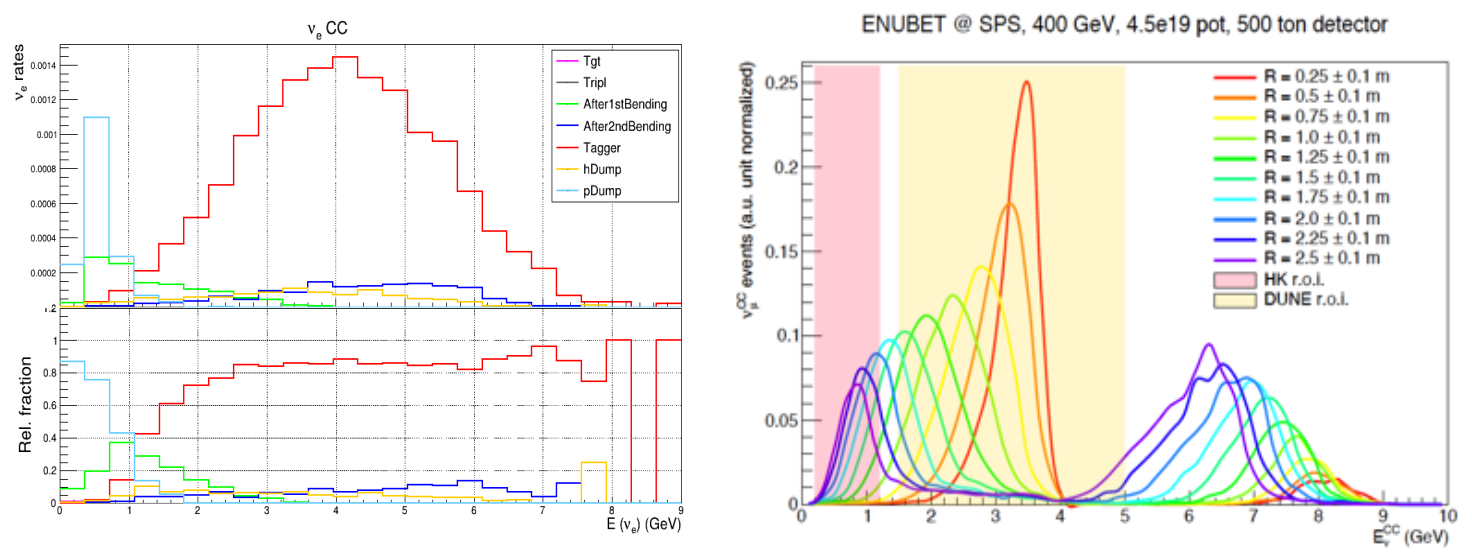

Figure 1: Left: energy spectrum (top) of the $v_{e}^{C C}$ interactions in the neutrino detector (see text). Each color corresponds to a neutrino contribution produced within a given part of the facility. The red line shows neutrinos produced within the tunnel. Relative fraction (bottom) of each category to the total $v_{e}^{C C}$ rate. Right: $v_{\mu}$ energy spectra for kaons and pions for different values of $\mathrm{R}$ (see text).

\section{Decay tunnel instrumentation and prototypes}

The decay tunnel of ENUBET will be instrumented with a sampling calorimeter for the positron and muon tagging and $e^{+} / \pi^{+} / \mu$ separation. The basic calorimeter module is a Lateral Compact Module (LCM) with longitudinal segmentation. Three radial layers of LCM instrument the tunnel walls. WLS fibers are used for the light collection, readout through SiPMs. Rings formed by doublets of scintillator tiles, installed in the inner tunnel surface, play the role of photon veto, allowing $e^{+} / \pi^{0}$ separation, as well as absolute event timing. This system is named t0-layer.

Tests were performed in 2016-2018 at the CERN East Experimental Area with prototypes built to validate the physics performance, in particular the $e^{+} / \pi^{+}$and $e^{+} / \pi^{0}$ separation capabilities [5-8]. Results of the beamline tests show an electron energy resolution of $\sigma_{E} / E=17 \%$ at $1 \mathrm{GeV}$, where a $<25 \% / \sqrt{E(\mathrm{GeV})}$ energy resolution is required to disentangle $e^{+} / \pi^{+}$in the $1-3 \mathrm{GeV}$ energy range of interest [9]. The hadronic shower profile from pions is reproduced by simulation within $10 \%$, compatible within the uncertainty due to low-energy hadronic shower simulation. The analysis of the t0-layer data show a capability of selecting one m.i.p. signals with $87 \%$ of efficiency, and a two m.i.p. rejection efficiency of $89 \%$. These values correspond to a purity of $95 \%$. The estimated time resolution is $\sim 400 \mathrm{ps}$. A final demonstrator is being built and will be exposed to test beam in 2022 .

\section{Lepton identification performance and assessment of systematics}

A full GEANT4 simulation of the ENUBET facility as been implemented. The simulation includes particle propagation and decay from transfer line to the detector, hit level detector response and a simplified approach to take into account pile-up effects. A realistic treatment of the pile-up is being pursued with the implementation of the waveform simulation response of the detector. The GEANT4 simulation has been validated with data from the prototype tests at CERN during 2016-2018. 
The analysis chain consists in an event builder algorithm and a signal/background separation step based on neural network. The achieved PID performance are: $\epsilon \sim 22 \%$ with a $S / N \sim 2$ for $K^{+} \rightarrow \pi^{0} e^{+} v_{e}$ and $\epsilon \sim 34 \%$ with a $S / N \sim 6$ for $K^{+} \rightarrow \mu^{+} v_{\mu}$ signal events. Ongoing studies are devoted to the development of an instrumented hadron dump for the identification of muons from pion decays.
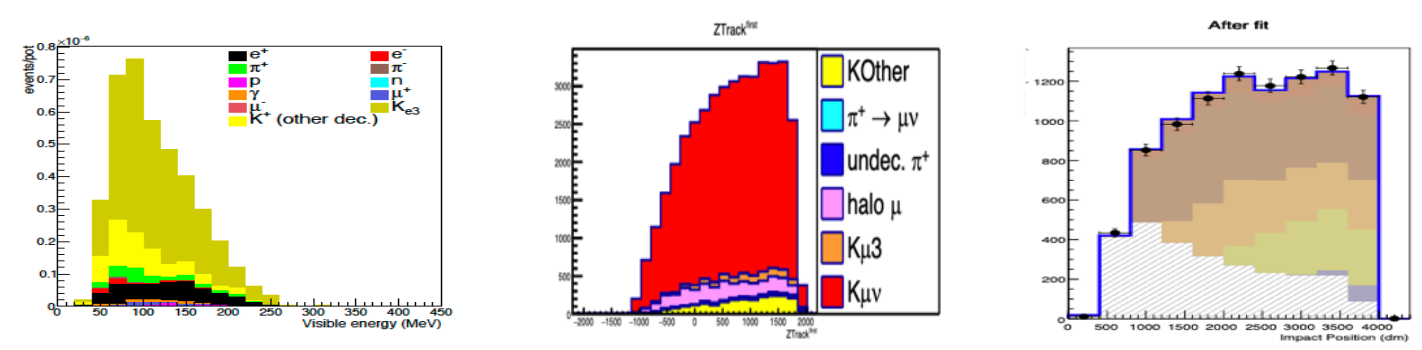

Figure 2: Visible energy of $K^{+} \rightarrow \pi^{0} e^{+} v_{e}$ (left) and impact point along calorimeter wall, Z, of $K^{+} \rightarrow \mu^{+} v_{\mu}$ (middle) reconstructed events after the application of the Neural Network. Right: example of one of the toy-MC fit with signal templates (coloured) and background template (dashed) for $K^{+} \rightarrow \mu^{+} v_{\mu}$ events along Z. Coloured templates correspond to different neutrino energies, providing energy dependent corrections on the neutrino flux.

To assess neutrino flux systematics, the relation between leptons measured in the calorimeter and neutrino flux at the detector is exploited. A signal plus background model is built, including a priori hadroproduction (HP) and TL related systematic uncertainties. Toy-MC experiments are produced and fitted to study the a posteriori systematic uncertainty and asses the corresponding uncertainty reduction. Eventually, the a postetiori systematic error is propagated to the neutrino flux by reweighting the simulation. Currently, tests of the procedure have been performed exploiting a toy HP model and mock kinematic observables. The next step is to build a model based on real HP data and observable templates from the facility simulation.

\section{References}

[1] A. Berra et al., CERN-SPSC-2016-036, SPSC-EOI-014, Geneva, 2016.

[2] F. Acerbi et al., CERN-SPSC-2018-034, SPSC-I-248, Geneva, 2018.

[3] A. Longhin, L. Ludovici and F. Terranova, Eur. Phys. J. C 75 (2015) 155.

[4] T. J. Roberts et al., Proc. EPAC'08 (2008) 2776-2779.

[5] A. Berra et al., Nucl. Instrum. Meth. A 830 (2016) 345.

[6] G. Ballerini et al., JINST 13 (2018) P01028.

[7] F. Acerbi et al., JINST 15 (2020) P08001.

[8] M. Pozzato et al., Nucl. Instrum. Meth. A 983 (2020) 164482.

[9] F. Pupilli et al., Positron identification in the ENUBET instrumented decay tunnel, PoS NEUTEL2017 (2018) 078. 